\title{
Quantum Chemical Investigation on Hydrolysis of Osmium(III) Anticancer Drug, (ImH)[trans-OsCl4(DMSO)(Im)] (Im=imidazole, Os-NAMI-A) and its Interaction with DNA
}

\author{
Paritosh Mondal $^{1}$, Amit Pradhan ${ }^{1}$, and Abhijit Shyam ${ }^{1}$ \\ ${ }^{1}$ Assam University
}

May 20, 2020

\begin{abstract}
Osmium analogue to ruthenium anticancer drug NAMI-A; (ImH)[trans-OsCl4(DMSO)(Im)] (Im=imidazole, DMSO=dimethyl sulfoxide) (Os-NAMI-A) shows a three-fold higher activity in colon carcinoma. Hydrolysis mechanism of Os-NAMI-A has been investigated using density functional theory (DFT) in combination with CPCM solvation model. Calculated activation free energy values for the first chloro ligand hydrolysis in the gaseous and aqueous medium are found to be $\Delta \mathrm{Gg}=31.79$ and $\Delta \mathrm{Gaq}=28.72 \mathrm{kcal} / \mathrm{mol}$, respectively. While, activation free energy for the second cis chloro ligand hydrolysis calculated in the gas and solvent phases are observed to be significantly lower $(\Delta \mathrm{Gg}=29.12$ and $\Delta \mathrm{Gaq}=22.61 \mathrm{kcal} / \mathrm{mol})$, suggesting enhanced feasibility of second hydrolysis. However, hydrolysis of DMSO ligand in the formation of cis- $[\mathrm{OsCl} 2(\mathrm{H} 2 \mathrm{O}) 3(\mathrm{Im})]+(\mathrm{P}-3 \mathrm{cis})$ is found to be thermodynamically preferred in aqueous medium $(19.49 \mathrm{kcal} / \mathrm{mol})$ with rate constant value of $3.20 \times 10-2 \mathrm{~s}-1$. In addition, molecular docking simulation reveals that cis-diaquated Os-NAMI-A (P-2cis) interacts with DNA (PDB ID: 1pgc) more effectively having binding energy $-5.63 \mathrm{kcal} / \mathrm{mol}$. Therefore, results of this investigation may lead us to understand the solution behaviour of osmium azole complexes as well as their mode of interaction with biomolecules which in return helps in potential anticancer drug designing.
\end{abstract}

\section{Hosted file}

Manuscript.doc available at https://authorea.com/users/324339/articles/452573-quantumchemical-investigation-on-hydrolysis-of-osmium-iii-anticancer-drug-imh-trans-oscl4-dmsoim-im-imidazole-os-nami-a-and-its-interaction-with-dna 